\title{
Improving Performance Analysis Of Routing Efficiency In Wireless Sensor Networks Using Greedy Algorithm
}

\author{
Dr. VUDA SREENIVASARAO ${ }^{1}$ \\ Professor, Dept. of CIT \\ Defence University College, \\ Deberezeit. Ethiopia.
}

\author{
CHANDRA SRINIVAS POTLURI ${ }^{2}$ \\ Asst Professor, Dept. of CSE \\ Aurora Sci Tech\& Research Academy \\ Hyderabad, India.
}

\author{
SREEDEVI KADIYALA ${ }^{3}$ \\ Lecturer, Dept. of CSE \\ Jigjiga University, Jigjiga. \\ Ethiopia
}

\author{
Capt. GENETU YOHANNES ${ }^{4}$ \\ HOD, Dept. of CIT \\ Defence University College, \\ Deberezeit,Ethiopia.
}

\begin{abstract}
The void problem causing the routing failure is the main challenge of the greedy routing in the wireless sensor networks. The current research work still cannot fully deal with the void problem since the excessive control overheads should be consumed so as to guarantee the delivery of packets. In this paper the solution to the void problem is taken up as the issue. This situation exists in the currently existing greedy routing algorithms in wireless sensor networks. The GAR protocol is a new protocol proposed here to guarantee the delivery of packets and excessive consumption of control overheads is resolved.
\end{abstract}

Keywords: Greedy routing; void problem; localized algorithm; wireless sensor network.

\section{INTRODUCTION}

Wireless sensor networks consist of a large number of inexpensive sensor nodes distributed in environment uniformly, having limited energy, therefore, in the most cases, nodes communicate with central node via their neighbours. On the other hand, an optimal route must be selected because there are different routes to central node from any other nodes. On the other hand, frequent use of one route results in reduction of energy of sensors located on that route and, ultimately, in sensors destruction. For solving this problem, we can consider a wireless sensor network as a graph in the nodes (hosts) which are the sensors and edges show the links between sensors.

Wireless sensor networks are currently using in different fields of research in both academic and industry. A wireless sensor networks is composed of a large number of nodes that are choose randomly dispersed over some of the interest area. Here all nodes in a wireless networks not communicate directly, so a multi hop routing protocol concept is required. Most of the routing protocols in wireless networks have been designed for networks of some number of hundreds of nodes and do not scale to networks with thousands of nodes.
Sensors combined in to machinery, environment, structures, coupled with the effective delivery of sensed information, could provide best benefits to society. The wireless sensor is networked and scalable, consumes very little power, it is software programmable and smart, it is good capable of speed data acquisition, reliable and accurate over the long term, costs little to purchase and install, and requires no real maintenance. This capability is enabling networks of very low cost sensors that are able to communicate with each other using low power wireless data routing protocols.

A wireless sensor network generally consists of a base station can be communicate with a number of wireless sensors via a radio link. Data is collected at the wireless sensor node, compressed and transmitted to the gateway directly or, if required, uses other wireless sensor nodes to forward data to the gateway. The transmitted data is presented to the system by the base station connection. A wireless sensor network (WSN) consists of sensor nodes ( $\mathrm{SNs}$ ) with wireless communication capabilities for specific sensing tasks.

Due to the limited available resources, efficient design of localized multi hope routing protocols becomes a crucial subject within the WSNs. How to guarantee delivery of packets is considered an important issue for the localized routing algorithms. The well-known greedy forwarding (GF) algorithm is considered a superior scheme with its low routing overheads. However, the void problem, which makes the GF technique unable to find its next closer hop to the destination, will cause the GF algorithm failing to guarantee the delivery of data packets.

Next generation environments represent the next evolutionary development step in utilities, building, home, industrial, shipboard, and transportation systems automation. Sensor network data comes from multiple sensors of different modalities in distributed in different locations. 
Routing algorithms are proposed to either resolve or reduce the void problem, which can be classified into non-graph-based and graph-based schemes. In the non-graph- based algorithms, the intuitive schemes as proposed in construct a two-hop neighbor table for implementing the GF algorithm. The network flooding mechanism is adopted within the GRA and PSR schemes while the void problem occurs. There also exist routing protocols that adopt the backtracking method at the occurrence of the network holes (such as GEDIR, , DFS , and SPEED ). The routing schemes as proposed by ARP and LFR memorize the routing path after the void problem takes place. Moreover, other routing protocols (such as PAGER NEAR and YAGR) propagate and update the information of the observed void node in order to reduce the probability of encountering the void problem. By exploiting these routing algorithms, however, the void problem can only be either 1) partially alleviated or 2) resolved with considerable routing overheads and significant converging time.

Several routing algorithms are proposed to either resolve or reduce the void problem, which can be classified into nongraph-based and graph-based schemes. In the non-graph- based algorithms [3], [4], [5], [6], [7], [8], [9], [10], [11], the intuitive schemes as proposed in [3] construct a two-hop neighbor table for implementing the GF algorithm. The network flooding mechanism is adopted within the GRA [4] and PSR schemes while the void problem occurs. There also exist routing protocols that adopt the backtracking method at the occurrence of the network holes (such as GEDIR, [3], DFS [5], and SPEED [6]). The routing schemes as proposed by ARP and LFR memorize the routing path after the void problem takes place. Moreover, other routing protocols (such as PAGER [7], NEAR [8], DUA [9], INF [10], and YAGR [11]) propagate and update the information of the observed void node in order to reduce the probability of encountering the void problem. By exploiting these routing algorithms, however, the void problem can only be either 1) partially alleviated or 2) resolved with considerable routing overheads and significant converging time. The Gabriel graph (GG) and the relative neighborhood graph (RNG) are the two commonly used localized planarization techniques that abandon some communication links from the UDG for achieving the planar graph. Nevertheless, the usage of the GG and RNG a graph has significant pitfalls due to the removal of critical communication links, leading to longer routing paths to the destination.

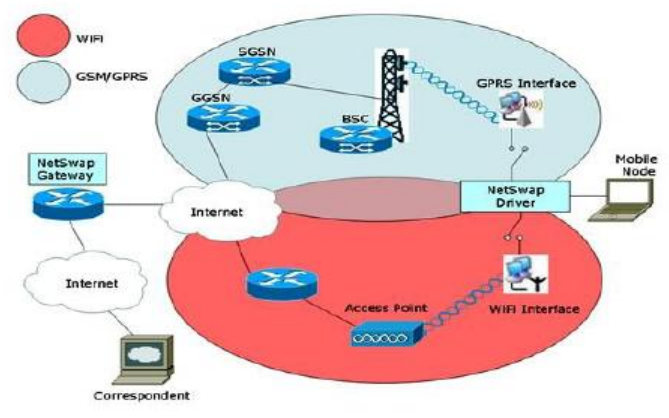

Figure 1. GPRS and Wi-Fi Interface

In figure 1 the working mechanism for GPSR and $\mathrm{Wi}-\mathrm{Fi}$ interface has been shown. The representative planar graph- based GPSR scheme cannot forward the packets from NV to NA directly since both the GG and the RNG planarization rules abandon the communication link from NV to NA. Considering the GG planarization rule for example, the communication link from NV.

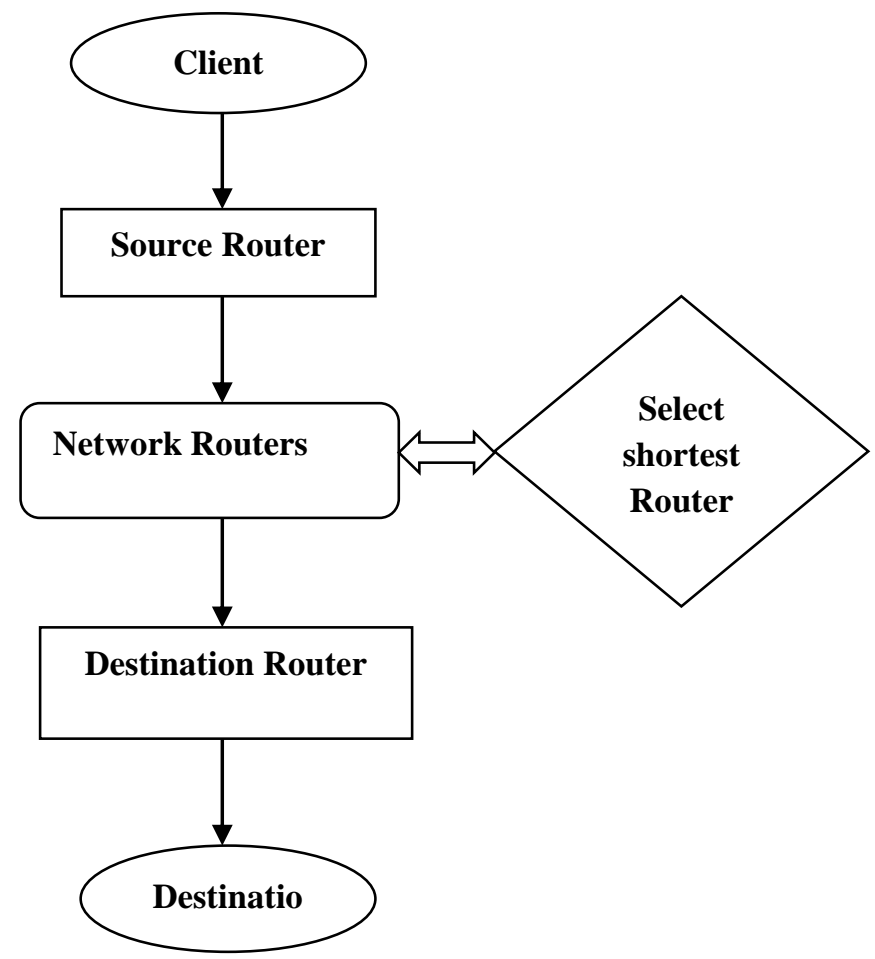

Figure 2. process of routers.

\section{RELATED WORK}

\section{A. Routing Algorithm:}

Let $\mathrm{N}$ is the number of nodes in the ad hoc network under consideration, Let $\mathrm{A}$ be the source node and $\mathrm{Z}$ be the destination node, $\mathrm{L}[\mathrm{X}]$ be the neighbor list of some arbitrary node X.

E num power status $=\{$ very low, low, medium, high, very high\};

hop count $=0$;

current node $=\mathrm{A}$

path $=$ 'A';

do

\{

If $\mathrm{Z} \hat{\mathrm{I}} \mathrm{L}$ [current node] /* If $\mathrm{Z}$ is the immediate neighbor */

$\{/ *$ Route found $* /$

Path= Path + 'Z';

Current node $=\mathrm{Z} ; / *$ destination reached $* /$

hop_count ++ ;

route $=$ path; 
Return route and hop_count;

Exit (0); /*Come out of the program*/

\}

else $/ * \mathrm{Z}$ is not the immediate neighbour*/

\{

$\mathrm{T}=0 ; l^{*}$ Number of neighbors whose $\mathrm{Z}$ is a immediateneighbor */

neighb_list=L[Current node]

while (neighb_list != empty)

\{ Remove first element of neighb_list, say X.

Send unicast message to $X$ to enquire about number of its neighbors N, Power Status

$\mathrm{P}$ and Ans to know if $\mathrm{Z}$ is a immediate neighbor of Current node;

Read and Record (node_id, P, N, Ans);

If (Ans="Y") T++;

\};

If $(\mathrm{T}==0) \mathrm{K}=1$; /* no one has information about the destination node $\mathrm{Z}^{*} /$

if $(\mathrm{T}==1) \mathrm{K}=2 ; / *$ only one node has information about the destination node $\mathrm{Z}^{* /}$

if $(\mathrm{T}>=1) \mathrm{K}=3 ; / *$ more then one node has information about the dhestination node $\mathrm{Z} * /$

Switch (K)

\{

Case 1: /* there is no neighbor node with $\mathrm{Z}$ as its immediate neighbor $* /$

For each neighbor of current node

Check (node id, P, N, ANS)

If $(\mathrm{P}=$ medium $\| \mathrm{P}=$ High $\| \mathrm{P}=$ Very high)

\{

Add node to the probable list;

For every node i belonging to probable list, find suitability index $\mathrm{Su}[\mathrm{i}]$.

Let $\mathrm{X}$ be node with highest suitability index.

$\mathrm{Su}=$ ord(power status) $-\mathrm{N} / \mathrm{C}$;

Choose $\mathrm{X}$ as the next node in route.

hop_count ++;

current node $=\mathrm{X}$;

path =path + 'X';

\}
Break;

Case 2: /* there is only one neighbor with $\mathrm{Z}$ as the immediate neighbor */

find record with (Ans= "Y");

Let this node be $\mathrm{X}$;

path=path +'X-Z';

Route $=$ path;

hop_count $=$ hop_count +2 ;

Return route and hop_count;

Exit (0);

Break;

Case 3: $/^{*}$ there is more than one neighbours with $\mathrm{Z}$ as the immediate neighbour" */

find records with (Ans= "Y");

Let these node be $\mathrm{X} 1, \mathrm{X} 2, \ldots \ldots \ldots . \mathrm{Xk}$;

For each node Xi to Xk find suitability index $\mathrm{Su}$.

$\mathrm{Su}=$ ord(power status) $-\mathrm{N} / \mathrm{C}$;

Choose the node with highest suitability index.

Let the node be $\mathrm{Y}$.

path=path +'Y-Z';

route $=$ path;

hop count=hop_count +2 ;

Return route and hop_count;;

Exit (0);

Break;

\}

\section{RESULTS}

Giving the Destination address in the source form:

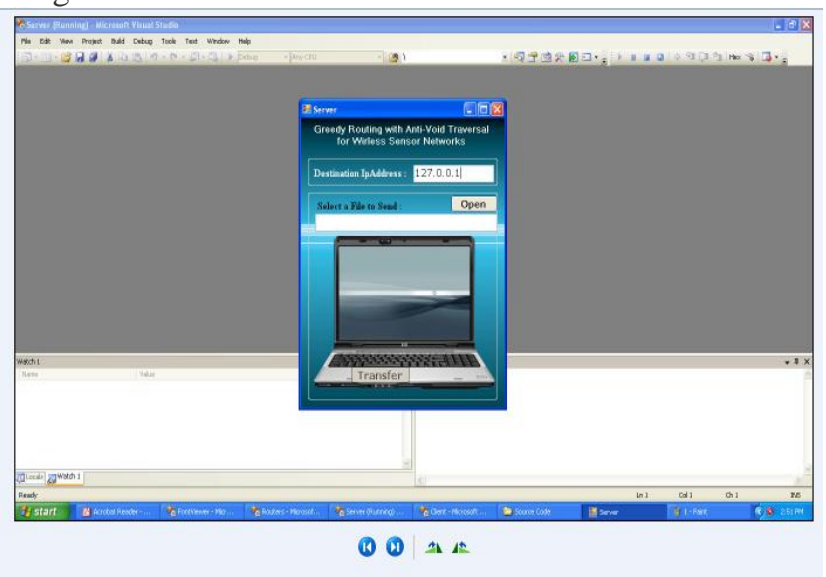

Figure 3. specify the destination IP address.

Server with selected file and destination address 


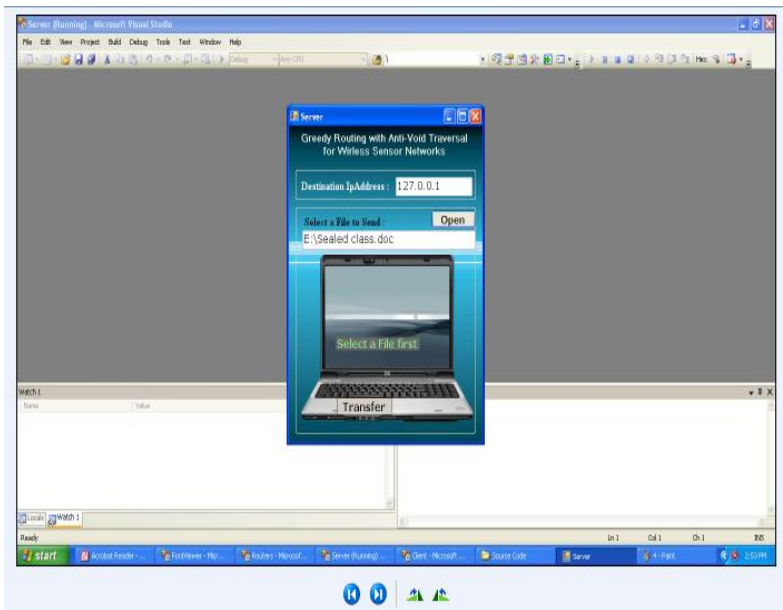

Figure 4. Destination IP address and a file to send.

Router Form to select the destination node

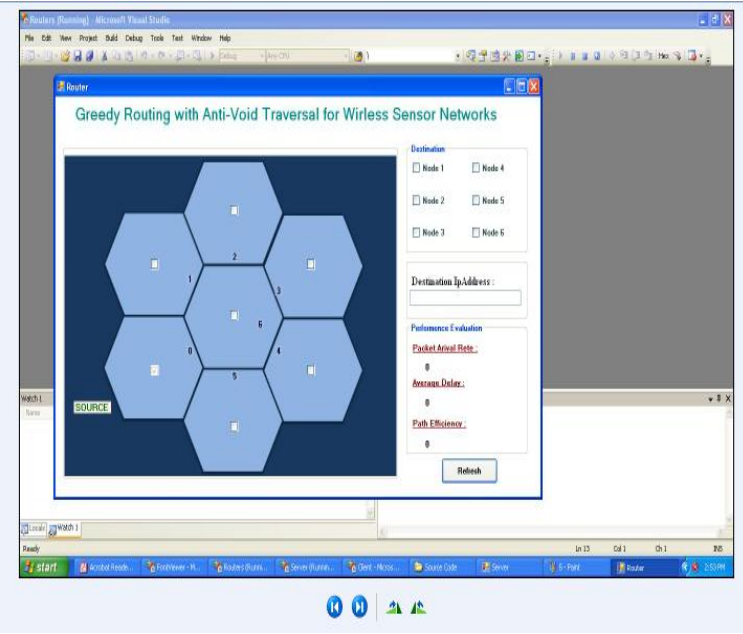

Figure 5. seven nodes selecting ' 0 ' as Source node.

Selecting destination node

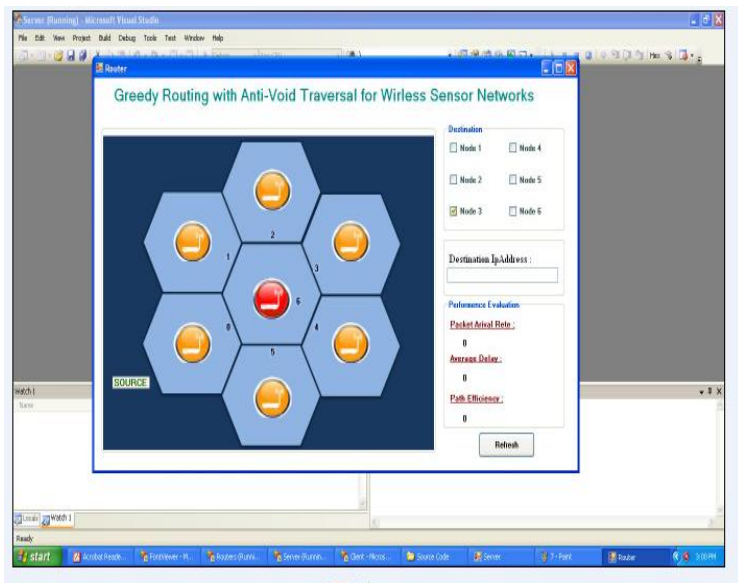

(1) (1) $4 \mathbb{2}$

Figure 6. the node 3 as destination node.
Screen showing the data transfer through routing path

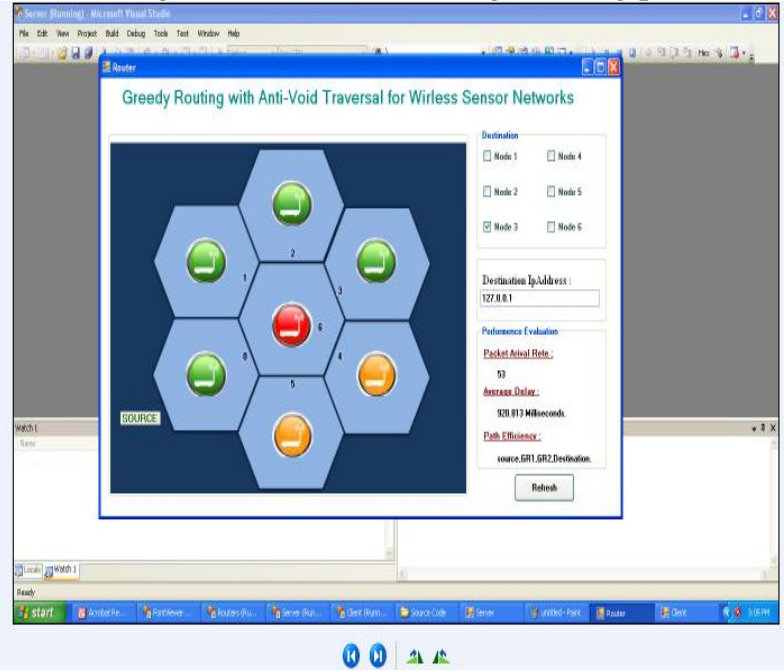

Figure 7. the transmission of file from one node to another node with green color. i.e. node 3 .

The file received by the destination

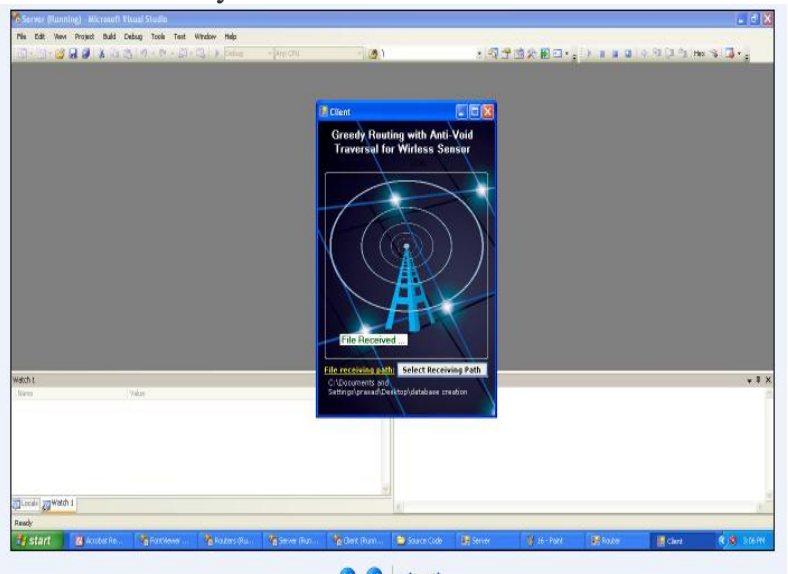

Figure 8. The successful transfer of file can be seen by observing "File Received" tab.

\section{CONCLUSION}

In this paper, a UDG based GAR protocol is proposed to solve the some void problems by using conventional GF algorithm. The RUT scheme is adopted with in the GAR protocol solve by using the boundary finding problem, results can be delivery of data packets under UDG networks. The correctness of the RUT scheme and the GAR algorithm is properly proven.

The performance of GAR Protocol is evaluated and compared with existing localized routing algorithms via simulations. The simulation study shows that the proposed GAR algorithm can guarantee the delivery of data packets with reduced communication overhead under different network scenarios. 


\section{REFERENCES}

[1] An efficient routing method for Ad hoc Networks by Mohinder Gupta

[2] Realization of Greedy Anti-Void Routing Protocol for Wireless Sensor Networks by Wen-Jiunn Liu and Kai-Ten Feng.

[3] B.Chella prabha, B.Anuradha,V.Kanimozhi, M.Dhivya "Enhanced Greedy Routing with Anti-Void Traversal for Wireless Sensor Networks" International journal of computing Applications in Engineering Sciences, Vol 1, Issue 1,March2011,pp- 18-22.

[4] Next century challenges: Scalable coordination in sensor NetworksD.Estrin, R.Govindan, J.Heidemann, and S.Kumar, ACM-PP-263270,Aug 1999.

[5] Data Communications and Networking, by Behrouz A Forouzan.

[6] Computer Networking: A Top-Down Approach, by James F. Kurose.

[7] Operating System Concepts, by Abraham Silberschatz.

[8] A Novel Routing Strategy For Ad Hoc Networks With Selfish Nodes by Shailender Gupta Chander Kumar,IJEECS.

[9] B.Chella prabha, B.Anuradha, V.Kanimozhi, M.Dhivya, "Enhanced Greedy Routing with Anti-Void Traversal for Wireless Sensor Networks", Journal of Computer Applications in Engineering Sciences (IJCAES) VOL I, ISSUE I, MARCH 2011.

\section{AUTHORS BIBLIOGRAPHY}

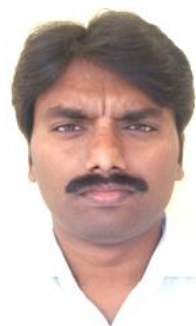

Dr. VUDASREENIVASARAO received his M.Tech degree in computer science and engineering from Sathyabama University from 2007.He received $\mathrm{PhD}$ degree in computer science and engineering from Singhania University from 2010. Currently working as Professor in the department of Computer and Information Technology, Defence University College, Deberezeit, Ethiopia. His main research interests are Data mining, Fuzzy logic and Network Security. He has got 11 years of teaching experience.
He has published 26 research papers in various international journals and one Springer international conference paper. He has 38 Editorial Board / Reviewers memberships in various international journals. $\mathrm{He}$ is a life member of various professional societies like IEEE, ACM, MAIRCC, MCSI, SMIACSIT, MIAENG, MCSTA and MISTE.

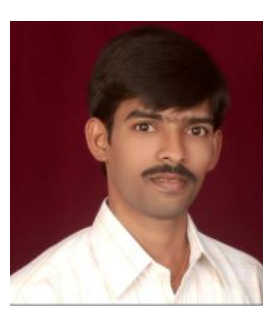

CHANDRA SRINIVAS POTLURI received his M.Tech degree in Computer science from JNT University. Currently working as Assistant Professor in Aurora Scientific Technological and Research Academy, Hyderabad, India. His main research interests are Data Mining \& Mobile Computing. He has got 5 years of teaching experience \& 4 years of Industrial experience worked in Satyam, Hyderabad, India.

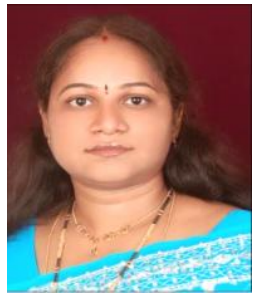

SREEDEVI KADIYALA received her M.Tech degree in Computer Science and Engineering from JNT University and M.Phil degree in Computer Science and Engineering from Bharathiar University. Currently working as a Lecturer in the department of CSE at Jigjiga University, Jigjiga, Ethiopia. Her main research interests are Data Mining and Data Ware housing, Network Security. She has got 9 years of teaching experience. She has published two papers in IJAST and IJCIS

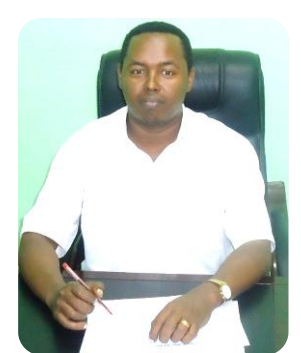

Capt. GENETU YOHANNES received his M.Tech degree in Computer Science \& Engineering from Defence Institute of Advanced Technology, Pune, in Year 2011. Currently working as HOD in the Department of Computer and Information Technology, Defence University College, Deberezeit, Ethiopia. He has got 7 years of teaching experience. His main research interests are Data Mining and Wireless Networks. 